\title{
Sex differences in intensive care unit admissions in Australia and New Zealand
}

Lucy Modra, David Pilcher, Michael Bailey and Rinaldo Bellomo

More men than women are admitted to intensive care units (ICUs) worldwide. ${ }^{1-6}$ In studies of sex differences in ICU admissions from North America, the United Kingdom and Europe, the percentage of female patients ranges from $35 \%$ to $45 \% .^{1-3,5-10}$ This simple observation likely reflects complex sex differences in the incidence, presentation and management of critical illness.

Previous studies focused on quantifying sex differences in illness severity and outcomes from ICU admission, with conflicting results. ${ }^{1,3,5-9}$ These studies highlight two important features of the sex imbalance in ICU admissions that warrant further investigation.

First, the sex imbalance is not evenly distributed across diagnostic categories. 5,8,9 Some critical illnesses have a marked male predominance; for example, trauma, cardiac surgery and aortic dissection. ${ }^{10-13}$ Other critical illnesses, such as subarachnoid haemorrhage and asthma, are more common in women. ${ }^{14,15}$ This raises a question: what is the relative contribution of different disease categories to the overall sex balance in ICU admissions?

Second, there is a wide variation in the reported percentage of female ICU patients in studies performed at different times in different regions of the world. Therefore, it is unclear if this variation is due to differences in casemix, differences in sociocultural context or changes over time.

This study examines these issues by systematically describing the sex balance in ICU admissions in Australia and New Zealand. We used the Australian and New Zealand Intensive Care Society (ANZICS) Centre for Resource and Outcome Evaluation (CORE) Adult Patient Database (APD). Our primary objective was to quantify the relative contribution of each major diagnostic category to the overall preponderance of men admitted to an ICU in Australia and New Zealand. Previous researchers have identified a sex imbalance across multiple diagnostic groups of critical illness $;, 6,10$ therefore, we hypothesised that a sex imbalance ( $<48 \%$ or $>52 \%$ women) existed across all major diagnostic categories of ICU admission. Secondary objectives were to identify the individual diagnoses with the most marked sex imbalance and to describe how the observed sex imbalance varied over time and with patient age.

\begin{abstract}
Introduction: Fewer women than men are admitted to intensive care units (ICUs) worldwide.

Objectives: To quantify the relative contribution of each major diagnostic category to the overall sex balance in ICU admissions in Australia and New Zealand, and to describe changes in the sex balance over time and with patient age. Methods: Retrospective cross-sectional study of Australian and New Zealand ICU admissions recorded in the Australian and New Zealand Intensive Care Society Adult Patient Database between 2005 and 2018. Multivariate logistic regression for the likelihood of female admission considered key explanatory variables: diagnostic category, patient age, admission year, geographic region, hospital type, and planned versus unplanned ICU admission.
\end{abstract}

Results: Overall, $42.3 \%$ of 1616856 Australian and New Zealand ICU patients were women $(99 \% \mathrm{Cl}, 42.2-42.4 \%)$. 247988 more men than women were admitted to an ICU during the 14-year study period. There was a sex imbalance in most diagnostic categories: less than $48 \%$ women in 15 of 23 diagnostic categories, and greater than $52 \%$ women in four diagnostic categories $(P<0.001)$. Admissions following cardiovascular surgery accounted for over half of the total sex imbalance. The percentage of ICU patients who are women increased linearly from $40.8 \%$ in 2005 to $43.6 \%$ in $2018\left(R^{2}=93.1 \% ; P<0.001\right)$. Compared with admission in 2005, the adjusted odds ratio for female admission in 2018 was $1.03(99 \% \mathrm{Cl}, 1.01-1.06)$.

Conclusion: There is a significant sex imbalance in ICU admissions in Australia and New Zealand, widespread across the diagnostic categories. Cardiovascular admissions contribute most to the observed preponderance of men. The proportion of female ICU patients is steadily increasing.

Crit Care Resusc 2021; 23 (1): 86-93 


\section{ORIGINAL ARTICLES}

\section{Methods}

Population

We undertook a retrospective cross-sectional study of ICU admissions in the ANZICS CORE APD between 1 January 2005 and 31 December 2018. The APD lists more than 2.4 million ICU episodes since 1993. Presently, over 180000 ICU admissions are submitted every year, estimated to account for $90 \%$ of ICU admissions in Australia and $70 \%$ of admissions in New Zealand. ${ }^{16}$ Sex is recorded as part of basic demographic data in this registry, taken from the patient's hospital record.

We excluded patients aged under 17 years; patients with missing diagnoses, sex classification or outcome; and repeat ICU admissions during the same hospital visit. Patients classified as "intersex" or "indeterminate sex" were excluded from the analysis because this sex classification was only introduced to the registry in 2017.

\section{Diagnostic categories}

We considered 23 diagnostic categories based on the Acute Physiology and Chronic Health Evaluation (APACHE) III-J categories used in the APD. ${ }^{16}$ Ten diagnostic groups had both postoperative and non-operative categories: cardiovascular, respiratory, gastrointestinal, neurological, trauma, metabolic, haematological, renal and genitourinary, female-specific, and musculoskeletal. The "sepsis" and "other" diagnoses had only non-operative categories, and "male-specific" diagnoses had a postoperative category only.

The female-specific diagnostic category included obstetric and gynaecological conditions, and the male-specific category included disorders of male urogenital tract. Postoperative admission was defined as admission to the ICU directly from the operating theatre or recovery after surgery.

\section{Statistical analysis}

Categorical variables are reported as counts with percentages to one decimal place. Given the size of the dataset, to increase robustness we set significance level at 0.01 ( $P<0.01$ indicates statistical significance) and reported 99\% confidence intervals $(\mathrm{Cls})$. The exact binomial test was used for comparisons of binary data and $\chi^{2}$ tests for other categorical variables. We defined a significant sex imbalance within diagnostic categories as less than $48 \%$ or greater than $52 \%$ of women. The relative contribution of each diagnostic category to the overall sex imbalance was calculated as the absolute difference between male and female admissions in the diagnostic category divided by the absolute difference between total male and total female admissions in the entire dataset.

Simple linear regression was used to assess the association between the percentage of female patients and year of admission. Multivariate logistic regression calculated the odds of an ICU admission being a female patient. Therefore, odds ratios (ORs) greater than one are associated with female ICU admission and ORs less than one are associated with male ICU admission. The multivariate model included the following categorical variables identified a priori as potentially associated with sex balance: APACHE III-J diagnostic category (postoperative and non-operative subcategories considered separately), patient age (10-year cohorts), admission year, hospital type (tertiary, metropolitan, rural/regional or private), geographic region (New Zealand or individual states and territories of Australia), and planned versus unplanned ICU admission.

Sex-specific diagnostic categories were excluded from the multivariate logistic regression. Year of admission was firstly modelled as a categorical variable and secondly modelled as a continuous variable to enable assessment of annual change over time. Results are reported as ORs $(99 \%$ $\mathrm{Cl}$ ), with a Wald $\chi^{2}$ statistic enabling a comparison of the relative significance of variables in the model.

Statistical analyses were performed with Stata 16.1 (StataCorp) and SAS 9.4 (SAS Institute).

\section{Ethics}

Ethics approval for this study was granted by the Alfred Hospital Human Research Ethics Committee (HREC 712/18).

\section{Results}

In the 14 years from 2005 to 2018, there were 1800521 ICU admissions in the ANZICS CORE APD. After exclusions, 1616856 patients were examined in this study (Online Appendix, figure 1). There were 326 patients excluded due to missing sex classification, and 111 patients were classified as intersex. Intersex patients composed less than $0.1 \%$ of admissions in 2017 (24/158 786) and 2018 (87/151 368).

Overall, women accounted for $42.3 \%(99 \% \mathrm{Cl}, 42.2-$ $42.4 \%$ ) of the 1616856 patients. There were 247988 more men than women in the dataset. This absolute difference in the number of men and women represents the total sex imbalance.

\section{Sex imbalance within diagnostic categories}

A sex imbalance was observed in most diagnostic categories (Table 1). Less than $48 \%$ of patients were women in 15 of 23 categories $(P<0.001$ for all) (Table 1$)$. More than $52 \%$ of patients were women in the two metabolic categories (postoperative and non-operative) and the two femalespecific categories (postoperative and non-operative) $(P<0.001$ for all). Four categories had similar numbers of men and women: the postoperative musculoskeletal, 
Table 1. Female intensive care unit (ICU) admissions by diagnostic category

\begin{tabular}{|c|c|c|c|c|}
\hline Diagnostic category & $\begin{array}{l}\text { Total admissions } \\
(n=1616856)\end{array}$ & $\begin{array}{c}\text { Female } \\
\text { admissions } \\
(n=684434)\end{array}$ & $\begin{array}{l}\text { Percentage } \\
\text { of female } \\
\text { admissions }\end{array}$ & $\begin{array}{l}\text { Odds ratio female } \\
\text { admission* } \\
(99 \% \mathrm{Cl})\end{array}$ \\
\hline \multicolumn{5}{|l|}{ Trauma } \\
\hline Non-operative & 53224 & 13249 & $24.9 \%$ & $0.33(0.26-0.41)$ \\
\hline Postoperative & 25916 & 7929 & $30.6 \%$ & $0.43(0.34-0.53)$ \\
\hline \multicolumn{5}{|l|}{ Cardiovascular } \\
\hline Non-operative & 120883 & 49404 & $40.9 \%$ & $0.68(0.55-0.85)$ \\
\hline Postoperative & 301806 & 82469 & $27.3 \%$ & $0.39(0.31-0.48)$ \\
\hline \multicolumn{5}{|l|}{ Renal and genitourinary } \\
\hline Non-operative & 20710 & 8505 & $41.1 \%$ & $0.69(0.56-0.86)$ \\
\hline Postoperative & 32330 & 10443 & $32.3 \%$ & $0.48(0.38-0.59)$ \\
\hline \multicolumn{5}{|l|}{ Respiratory } \\
\hline Non-operative & 171155 & 80545 & $47.1 \%$ & $0.88(0.71-1.09)$ \\
\hline Postoperative & 72705 & 27995 & $38.5 \%$ & $0.62(0.5-0.77)$ \\
\hline \multicolumn{5}{|l|}{ Gastrointestinal } \\
\hline Non-operative & 54498 & 22576 & $41.4 \%$ & $0.69(0.56-0.86)$ \\
\hline Postoperative & 215902 & 100739 & $46.7 \%$ & $0.86(0.69-1.06)$ \\
\hline Sepsis, non-operative & 103390 & 46654 & $45.1 \%$ & $0.82(0.66-1.02)$ \\
\hline \multicolumn{5}{|l|}{$\begin{array}{l}\text { Musculoskeletal, skin, and soft } \\
\text { tissue }\end{array}$} \\
\hline Non-operative & 3935 & 1712 & $43.5 \%$ & $0.75(0.60-0.95)$ \\
\hline Postoperative & 87327 & 43951 & $50.3 \%$ & $0.96(0.77-1.19)$ \\
\hline \multicolumn{5}{|l|}{ Neurological } \\
\hline Non-operative & 81241 & 36724 & $45.2 \%$ & $0.83(0.67-1.03)$ \\
\hline Postoperative & 112271 & 55292 & $49.2 \%$ & $0.96(0.77-1.19)$ \\
\hline Male-specific, postoperative & 4355 & 32 & $0.7 \%$ & na \\
\hline \multicolumn{5}{|l|}{ Haematological } \\
\hline Non-operative & 7182 & 3258 & $45.4 \%$ & $0.84(0.67-1.05)$ \\
\hline Postoperative & 578 & 292 & $50.5 \%$ & $1.0^{+}$ \\
\hline Other, non-operative & 9070 & 4651 & $51.3 \%$ & $1.01(0.81-1.26)$ \\
\hline \multicolumn{5}{|l|}{ Metabolic } \\
\hline Non-operative & 99302 & 53681 & $54.1 \%$ & $1.11(0.90-1.38)$ \\
\hline Postoperative & 12651 & 8050 & $63.6 \%$ & $1.73(1.39-2.16)$ \\
\hline \multicolumn{5}{|l|}{ Female-specific } \\
\hline Non-operative & 4000 & 3975 & $99.4 \%$ & na \\
\hline Postoperative & 22425 & 22308 & $99.5 \%$ & na \\
\hline Total & 1616856 & 684434 & $42.3 \%$ & \\
\hline
\end{tabular}

na $=$ not applicable. * Odds ratios adjusted for patient age, admission year, hospital type (tertiary, metropolitan, rural/regional or private), geographic region and planned versus unplanned ICU admission. Sex-specific diagnoses were excluded from the model $(n=1586076,41.5 \%$ women, after excluding 30780 admissions for sex-specific diagnoses). † Reference. 


\section{ORIGINAL ARTICLES}

Figure 1. Contribution to overall sex imbalance by diagnostic category

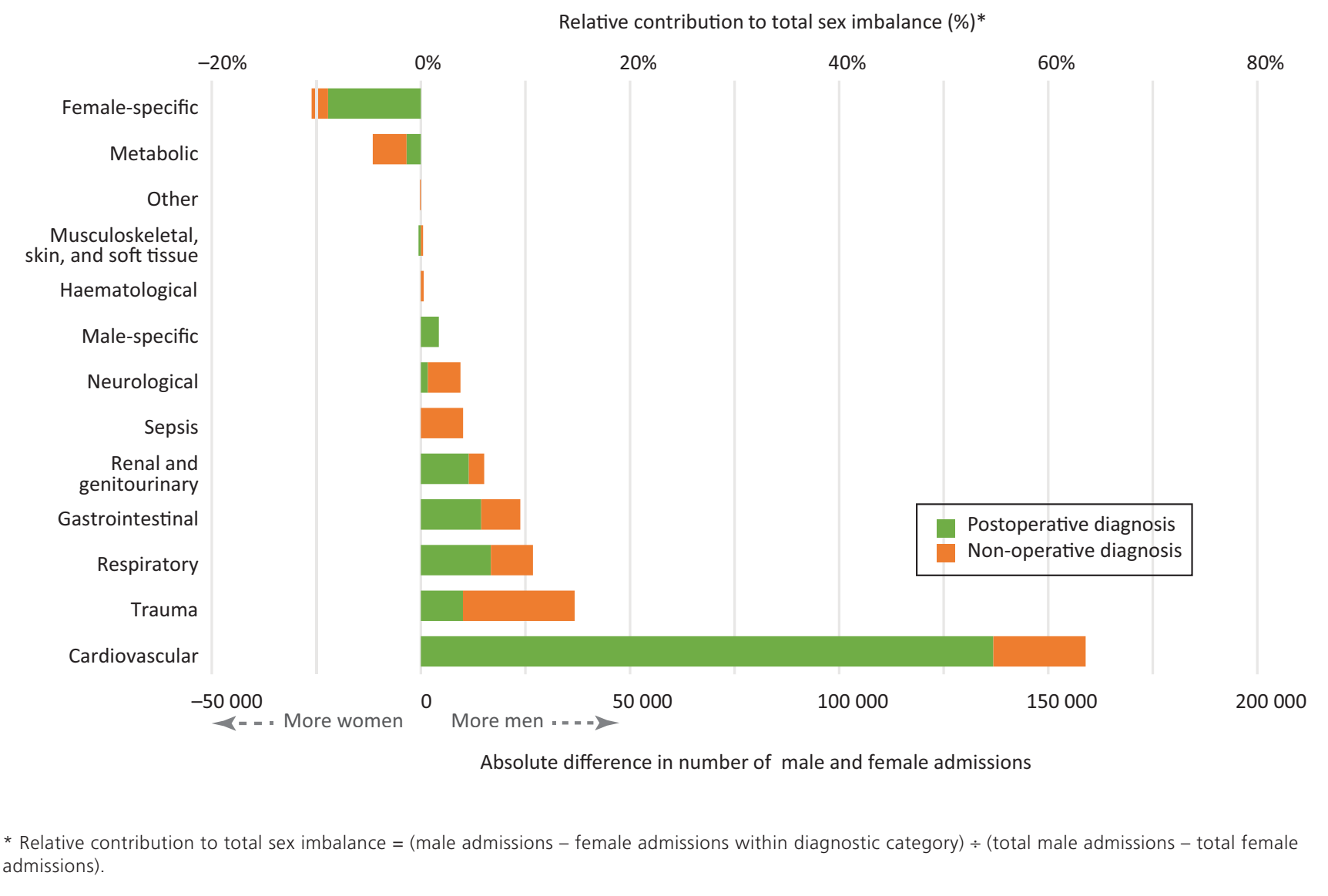

neurological and haematological categories and the nonoperative "other" diagnostic category.

The sex imbalance was not evenly distributed across diagnostic categories (Figure 1).

Cardiovascular diagnoses contributed the most to the overall sex imbalance in ICU admissions. Postoperative cardiovascular surgery admissions accounted for over half $(55.2 \%)$ of the total sex imbalance in the dataset; there were 136868 more men than women in this diagnostic category. After removing cardiovascular surgery admissions from the dataset, $45.8 \%$ of remaining patients were women (99\% Cl, 45.7-45.9\%).

Trauma, gastrointestinal and respiratory diagnoses all contributed substantially to the total sex imbalance, accounting for $14.8 \%, 10.8 \%$ and $9.6 \%$ of the total imbalance respectively (Figure 1). Sepsis diagnoses accounted for only $4.1 \%$ of the total sex imbalance: 10082 more men than women were admitted with sepsis.

The female-specific diagnostic category $(n=26425)$ had substantially more admissions than the corresponding male- specific diagnostic category $(n=4355)$. Aside from femalespecific diagnoses, the metabolic categories, including endocrine and thermal emergencies and electrolyte disturbances, had the highest percentage of female patients ( $55.1 \%$ overall).

\section{Individual diagnoses with the largest sex imbalance}

Aside from sex-specific diagnoses, the diagnoses with highest percentage of female patients were asthma (69.7\% women), endocrine surgery $(63.6 \%$ women) and subarachnoid haemorrhage (63.2\% women) (Online Appendix, table 3). The male-dominated diagnoses were all from the cardiovascular surgery and trauma categories, including endoluminal aortic repair (18.2\% women), cervical spine injury (18.3\%) and ruptured aortic aneurysm (18.7\% women).

\section{Change over time}

Over 14 years, the proportion of all ICU patients who were women increased linearly from $40.8 \%$ in 2005 to $43.6 \%$ 


\section{ORIGINAL ARTICLES}

in 2018 (Figure 2, A). Simple linear regression of the proportion of female ICU patients based on admission year revealed a strong linear association $\left(P<0.001 ; R^{2}=93.1 \%\right)$. There was an increase over time in the relative odds of an ICU admission being a female patient after adjustment in the multivariate model, with an annual change in the OR for female admission of 1.004 (99\% Cl, 1.003-1.005; $P<0.0001$ ) (Figure 2, B).

\section{Change with patient age}

There was a curvilinear relationship between the percentage of female admissions and patient age, with the highest percentage of female patients observed in the cohort aged $30-39$ years ( $51.2 \%$ women; $99 \% \mathrm{Cl}, 50.8-51.6 \%)$ and in the oldest cohort aged 80 years and over (46.9\% women; $99 \% \mathrm{Cl}, 46.6-47.1 \%)$. The cohort aged 60-69 years had the lowest percentage of female patients (37.6\% women;

Figure 2. (A) Percentage of female intensive care unit (ICU) admissions over time (unadjusted); (B) odds ratio of female ICU admission over time (adjusted)*

A

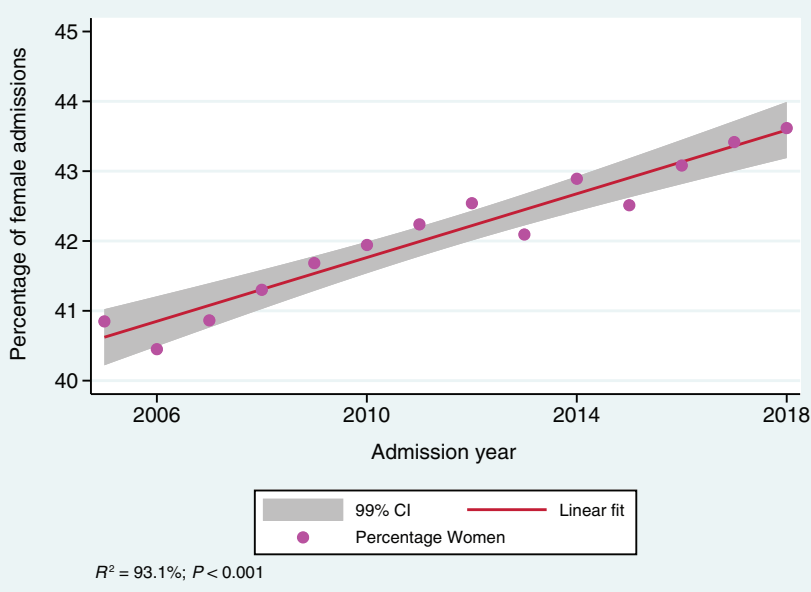

B

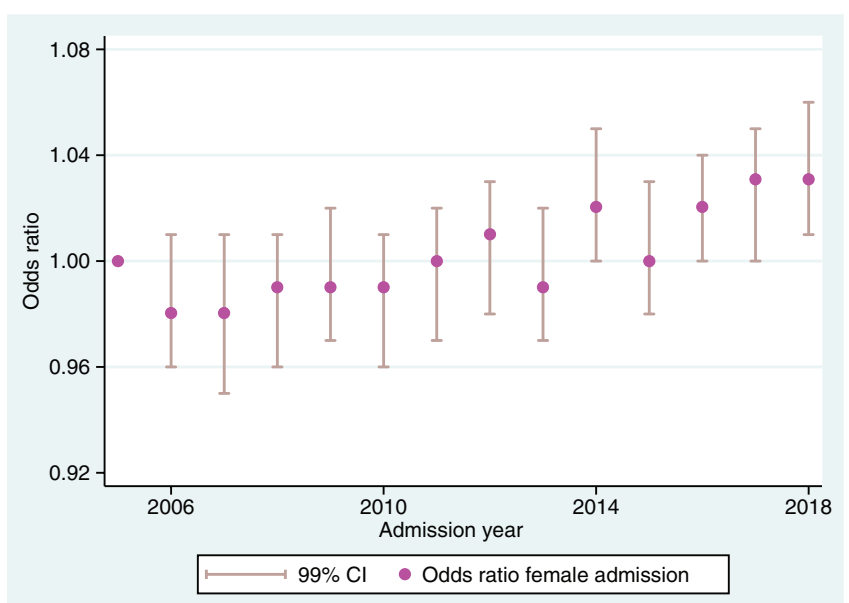

* Adjusted for Acute Physiology and Chronic Health Evaluation (APACHE) III-J diagnostic category, patient age, admission year, hospital type (tertiary, metropolitan, rural/regional or private), geographic region (New Zealand or state or territory of Australia) and planned versus unplanned ICU admission.

Figure 3. (A) Percentage of female admissions by age cohort (unadjusted); (B) odds ratio for female ICU admission by age cohort (adjusted*)

A

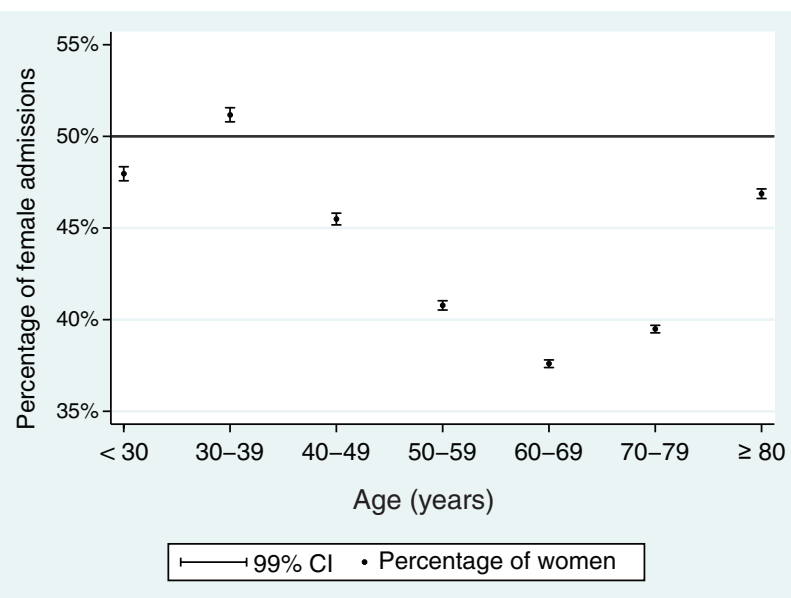

B

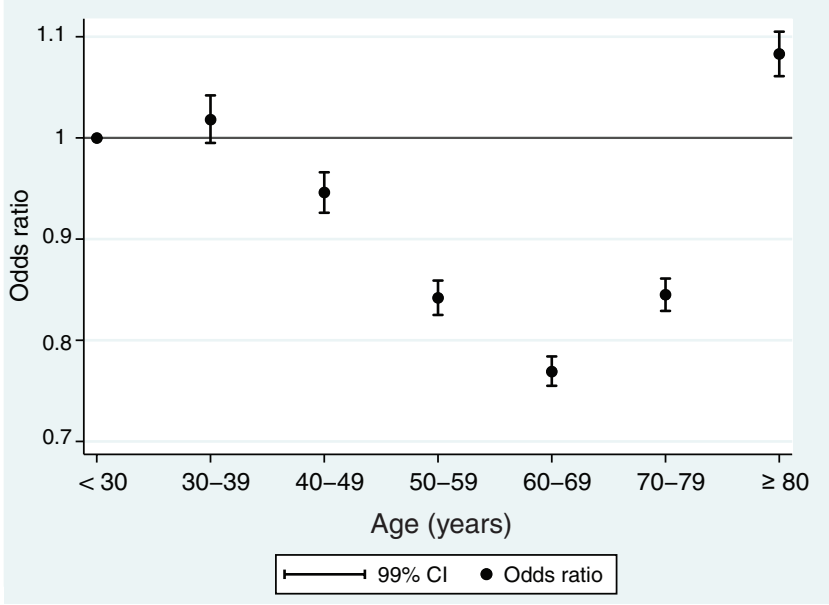

* Adjusted for Acute Physiology and Chronic Health Evaluation (APACHE) III-J diagnostic category, patient age, admission year, hospital type (tertiary, metropolitan, rural/regional or private), geographic region (New Zealand or state or territory of Australia) and planned versus unplanned ICU admission. 


\section{ORIGINAL ARTICLES}

$99 \% \mathrm{Cl}, 37.4-37.8 \%)$. This curvilinear association between age and sex balance persisted after adjusting for casemix variables including diagnostic category in the multivariate logistic regression (Figure 2, B). Compared with those aged less than 30 years, the $\mathrm{OR}$ for female admission in the cohort aged 60-69 was $0.77(99 \% \mathrm{Cl}, 0.76-0.78)$.

\section{Multivariate logistic regression}

The variable with the greatest impact on sex balance in our model was diagnostic category, followed by patient age, hospital type, geographic region and year of admission. Planned versus unplanned ICU admission status was not statistically significantly associated with sex balance (Online Appendix, table 2).

Compared with metropolitan hospitals, there was a decreased relative odds of female ICU admission at tertiary centres $(\mathrm{OR}, 0.88 ; 99 \% \mathrm{Cl}, 0.87-0.91)$, and increased relative odds of female ICU admission at private hospitals (OR, 1.03; 99\% Cl, 1.02-1.05). Several Australian regions had a significantly lower likelihood of female ICU admission compared with the reference region New Zealand, most notably Western Australia (OR, 0.88; $99 \% \mathrm{Cl}, 0.86-0.91)$ and the Northern Territory (OR, $0.91 ; 99 \% \mathrm{Cl}, 0.87-0.94)$.

\section{Discussion}

\section{Key findings}

In this comprehensive binational registry-based study we found a substantial sex imbalance in ICU admissions in Australia and New Zealand. Women made up $42.3 \%$ of all ICU admissions; nearly a quarter of a million more men than women were admitted to an ICU between 2005 and 2018. The sex imbalance was widespread across most diagnostic categories. Cardiovascular diagnoses were the single largest driver of the total sex imbalance.

The percentage of ICU patients who are women increased linearly over time. After adjustment for the pre-specified casemix variables, including diagnostic category, age and region, there was a small increase over time in likelihood of an ICU patient being female. Regarding age, the lowest percentage of female patients occurred in the cohorts aged 50-79 years, and this pattern persisted in the multivariate model. Admission diagnosis was more strongly associated with sex balance than other variables, including age and admission year.

\section{Relationship to previous studies}

The percentage of female ICU patients in this study (42.3\%) is in the middle of the range reported in previous studies of sex differences in ICU admissions from North America, the UK and Europe (35-45\% women). ${ }^{1-3,5,6,8,10}$ Most of these studies looked at a modest proportion of ICUs within one or two nations. ${ }^{1-3,5,6,8-10}$ Samuelsson and colleagues $^{7}$ examined a comprehensive multicentre cohort of 127254 ICU admissions in Sweden, of whom $43 \%$ were women. In contrast, our study included the majority of all ICU admissions in Australia and New Zealand across 14 years, with over five times the total number of admissions considered in the previous largest study.

Our study is the first to systematically describe sex balance across all diagnostic categories leading to ICU admission. Previous studies reported the casemix for male and female admissions separately rather than reporting sex balance within each diagnostic category. 1,3,7,9 Other studies examined sex differences within discrete critical illnesses including sepsis and cardiac arrest. 5,17-20

Our study demonstrates that sepsis - a diagnostic group in which sex differences are relatively well studied - only makes a modest contribution to the overall sex imbalance in ICU admissions. Moreover, the sex imbalance is actually widespread across most diagnostic categories, including diagnoses in which sex differences are not yet well studied.

The preponderance of men among cardiovascular admissions in this study is consistent with previous findings that men have a higher incidence of many cardiovascular diseases, including ischaemic heart disease and aortic disease. ${ }^{12,13,21,22}$ Studies from Europe and the United States found that between $23.5 \%$ and $36.5 \%$ of trauma patients admitted to the ICU were women and we found a similarly low percentage (26.8\%) of female trauma patients. $3,6,8,10$ In addition, this study found that trauma admissions made a modest contribution to the overall ICU sex imbalance because of the low number of trauma admissions overall.

Romo and colleagues reported an increase in the percentage of female ICU patients over two separate years, from $33.5 \%$ in 1983 to $38 \%$ in 1995.23 Our study also found an increasing percentage of female patients over time. Additionally, we reported a linear increase in the percentage of female patients over 14 consecutive years; this persisted after adjustment for other casemix factors. The change in sex balance is unlikely to be solely due to changing population demographics: over the study period, the percentage of women in the Australian population increased from $50.3 \%$ to $50.4 \%, 24,25$ and in the New Zealand population it decreased from $51.0 \%$ to $50.8 \% .^{26}$

Garland and colleagues ${ }^{2}$ reported that about $80 \%$ of the total sex imbalance in ICU admissions in Manitoba, Canada, could be attributed to the cohort aged $45-74$ years. ${ }^{2}$ Similarly, we found the most prominent sex imbalance in the cohort aged 50-79 years and this finding persisted after adjustment for diagnostic category and other casemix variables. This suggests a higher risk of critical illness per se among men in this age group, rather than reflecting 


\section{ORIGINAL ARTICLES}

the age-related incidence of specific disease leading to ICU admission (eg, coronary artery disease).

\section{Implications of study findings}

The finding that sex imbalance exists across most diagnostic categories leading to ICU admission implies a systemic difference in the way that men and women manifest, present with and/or are treated for critical illness resulting from a range of primary organ pathologies. This observation tends to support a broader approach to studying sex differences in critical illness.

Cardiovascular diagnoses were the single largest contributor to the total sex imbalance in ICU admissions, reflecting both the very low percentage of female patients within the cardiovascular diagnostic category and the very high number of cardiovascular admissions. The former can be attributed largely to sex differences in the incidence of cardiovascular disease. However, there are also differences in the way men and women with the same cardiovascular condition are evaluated and treated. For example, women are less likely than men to receive coronary interventions when presenting with acute coronary syndromes and cardiac arrest, and are less likely to be prescribed $\beta$-blockers and statins following myocardial infarction. $20,22,27$ Therefore, it is possible that systemic differences in the treatment of men and women with cardiovascular disease also contributed to the observed sex imbalance in ICU admissions.

The percentage of ICU patients who were women increased over time. This increase attenuated in the multivariate model, suggesting that it can be largely attributed to changes in ICU casemix. However, the small increase in adjusted odds of female admission over time suggests that the sex balance is influenced by variables not considered in the model, such as sociocultural factors.

\section{Strengths and weaknesses}

This is the largest study of sex differences in ICU admissions to date, drawing from a well established registry including well over $80 \%$ of all ICU admissions in Australia and New Zealand. ${ }^{16}$ It is the first to systematically describe the relative contribution of diagnostic groups to the sex imbalance in ICU admissions.

However, this initial descriptive study is limited in scope. We set out to systematically describe the phenomenon of sex imbalance in the ICU patient population particularly focusing on admission diagnosis and change over time and with patient age. We did not consider the denominator of all potential ICU admissions (including aged-based population sex ratios), nor sex differences in illness severity and outcomes; therefore, we cannot comment on equity of access to the ICU. It is possible that the sex imbalance described in this study represents fair and equitable access to the ICU.
We considered sex-specific diagnoses separately in order to assess their impact on the overall sex balance. However, we could not identify all sex-specific conditions because of incomplete diagnostic subcode data. Therefore, some malespecific conditions likely remained in the genitourinary and renal category; for example, cases of prostate cancer coded as "genitourinary neoplasm" rather than subcoded as "prostate cancer". This could have falsely inflated the sex imbalance within the renal and genitourinary category and decreased the size of the male-specific diagnostic category. In addition, there were some male patients with femaledesignated diagnoses and vice versa ( $<1 \%$ of each category). This could represent a coding error or patients whose selfidentified gender does not match their biological sex.

\section{Conclusion}

Substantially more men than women are admitted to an ICU in Australia and New Zealand and a sex imbalance occurs across most diagnostic categories leading to ICU admission. Diagnostic category is a strong predictor of sex balance in ICU admissions. Among the diagnostic categories, cardiovascular diagnoses contribute the most to the overall sex imbalance. The percentage of ICU patients who are women is increasing linearly over time. These findings support further research into sex differences in critical illness and equity of access to the ICU.

Acknowledgements: The authors and the ANZICS CORE Management Committee thank clinicians, data collectors and researchers from the contributing sites listed in the Online Appendix.

\section{Competing interests}

None declared.

\section{Author details}

Lucy Modra ${ }^{1,2}$

David Pilcher $3,4,5$

Michael Bailey ${ }^{4}$

Rinaldo Bellomo 1,2, 4, 6

1 Department of Intensive Care, Austin Health, Melbourne, VIC, Australia.

2 Centre for Integrated Critical Care, University of Melbourne, Melbourne, VIC, Australia.

3 Department of Intensive Care, Alfred Hospital, Melbourne, VIC, Australia.

4 Australia and New Zealand Intensive Care Research Centre, 


\section{ORIGINAL ARTICLES}

School of Public Health and Preventative Medicine, Monash University, Melbourne, VIC, Australia.

5 Australian and New Zealand Intensive Care Society (ANZICS), Centre for Outcome and Resource Evaluation, Melbourne, VIC, Australia.

6 Department of Intensive Care, Royal Melbourne Hospital, Melbourne, VIC, Australia.

Correspondence: lucy.modra@austin.org.au

\section{References}

1 Mahmood K, Eldeirawi K, Wahidi MM. Association of gender with outcomes in critically ill patients. Crit Care 2012; 16: R92.

2 Garland A, Olafson K, Ramsey CD, et al. Reassessing access to intensive care using an estimate of the population incidence of critical illness. Crit Care 2018; 22: 208.

3 Hollinger A, Gayat E, Feliot E, et al. Gender and survival of critically ill patients: results from the FROG-ICU study. Ann Intensive Care 2019; 9: 43.

4 Park J, Jeon K, Chung CR, et al. A nationwide analysis of intensive care unit admissions, 2009-2014 - the Korean ICU National Data (KIND) study. J Crit Care 2018; 44: 24-30.

5 Raine R, Goldfrad C, Rowan K, Black N. Influence of patient gender on admission to intensive care. J Epidemio/ Community Health 2002; 56: 418-23.

6 Reinikainen M, Niskanen M, UUsaro A, Ruokonen E. Impact of gender on treatment and outcome of ICU patients. Acta Anaesthesiol Scand 2005; 49: 984-90.

7 Samuelsson C, Sjoberg F, Karlstrom G, et al. Gender differences in outcome and use of resources do exist in Swedish intensive care, but to no advantage for women of premenopausal age. Crit Care 2015; 19: 129.

8 Valentin A, Jordan B, Lang T, et al. Gender-related differences in intensive care: a multiple-center cohort study of therapeutic interventions and outcome in critically ill patients. Crit Care Med 2003; 31: 1901-7.

9 Fowler RA, Sabur N, Li P, et al. Sex-and age-based differences in the delivery and outcomes of critical care. CMAJ 2007; 177: 1513-9.

10 Zettersten E, Jaderling G, Bell M, Larsson E. Sex and gender aspects on intensive care. A cohort study. J Crit Care 2020; 55: 22-7.

11 Schoeneberg C, Kauther MD, Hussmann B, et al. Genderspecific differences in severely injured patients between 2002 and 2011: data analysis with matched-pair analysis. Crit Care 2013; 17: R277.

12 Johnston A, Mesana TG, Lee DS, et al. Sex differences in longterm survival after major cardiac surgery: a population-based cohort study. J Am Heart Assoc 2019; 8: e013260.

13 Nienaber CA, Fattori R, Mehta RH, et al. Gender-related differences in acute aortic dissection. Circulation 2004; 109: 3014-21.

14 De Marchis GM, Schaad C, Fung C, et al. Gender-related differences in aneurysmal subarachnoid hemorrhage: a hospital based study. Clin Neurol Neurosurg 2017; 157: 82-7.

15 Gupta D, Keogh B, Chung KF, et al. Characteristics and outcome for admissions to adult, general critical care units with acute severe asthma: a secondary analysis of the ICNARC Case Mix Programme Database. Crit Care 2004; 8: R112-21.

16 Australian and New Zealand Intensive Care Society Centre for Outcome and Resource Evaluation. ANZICS CORE 2018 report. Melbourne: ANZICS, 2019. https://www.anzics.com. au/wp-content/uploads/2019/10/2018-ANZICS-CORE-Report. pdf (viewed Dec 2020).

17 Adrie C, Azoulay E, Francais A, et al. Influence of gender on the outcome of severe sepsis: a reappraisal. Chest 2007; 132: 1786-93.

18 Luethi N, Bailey M, Higgins A, et al. Gender differences in mortality and quality of life after septic shock: A post-hoc analysis of the ARISE study. J Crit Care 2020; 55: 177-83.

19 Bosson N, Kaji A, Fang A, et al. Sex differences in survival from out-of-hospital cardiac arrest in the era of regionalized systems and advanced post-resuscitation care. J Am Heart Assoc 2016; 5: e004131.

20 Kim LK, Looser P, Swaminathan RV, et al. Sex-based disparities in incidence, treatment, and outcomes of cardiac arrest in the United States, 2003-2012. J Am Heart Assoc 2016; 5: e003704.

21 Albrektsen G, Heuch I, Lochen ML, et al. Lifelong gender gap in risk of incident myocardial infarction: the Troms $\varnothing$ study. JAMA Intern Med 2016; 176: 1673-9.

22 Khan E, Brieger D, Amerena J, et al. Differences in management and outcomes for men and women with ST-elevation myocardial infarction. Med J Aust 2018; 209: 118-23.

23 Romo H, Kajdacsy-Balla Amaral AC, Vincent JL. Effect of patient sex on intensive care unit survival. Arch Intern Med 2004; 164: 61-5.

24 Australian Bureau of Statistics. Population by age and sex, Australia, 2006 [Cat. No. 3235.0]. Canberra: ABS, 2007. https:// www.abs.gov.au/ausstats/abs@.nsf/(viewed June 2020).

25 Australian Bureau of Statistics. Regional population by age and sex, Australia, 2018. Canberra: ABS, 2019. Https:// www.abs.gov.au/AUSSTATS/abs@.nsf/allprimarymainfeatures/151AA7593B394934CA2573210018DA4A?opendocument (viewed June 2020).

26 New Zealand Government, Stats NZ. Summary figures for the New Zealand population, 1991-2019. Https://www.stats.govt. nz/topics/population (viewed Mar 2020).

27 Dey S, Flather MD, Devlin G, et al. Sex-related differences in the presentation, treatment and outcomes among patients with acute coronary syndromes: the Global Registry of Acute Coronary Events. Heart 2009; 95: 20-6. 